\title{
Indiennes : un tissu à la conquête du monde
}

Nouveau Centre des indiennes au Musée national suisse - Château de Prangins

\section{Helen Bieri Thomson}

\section{(2) OpenEdition \\ 12 Journals}

Édition électronique

URL : http://journals.openedition.org/artefact/5948

DOI : $10.4000 /$ artefact. 5948

ISSN : 2606-9245

Éditeur :

Association Artefact. Techniques histoire et sciences humaines, Presses universitaires du Midi

\section{Édition imprimée}

Date de publication : 15 juillet 2020

Pagination : 279-284

ISBN : 978-2-8107-0691-4

ISSN : 2273-0753

\section{Référence électronique}

Helen Bieri Thomson, «Indiennes : un tissu à la conquête du monde », Artefact [En ligne], 12 | 2020, mis en ligne le 21 décembre 2020, consulté le 23 décembre 2020. URL : http://journals.openedition.org/ artefact/5948; DOI : https://doi.org/10.4000/artefact.5948

\section{(c) (i) (9)}

Artefact, Techniques, histoire et sciences humaines est mise à disposition selon les termes de la Licence Creative Commons Attribution - Pas d'Utilisation Commerciale - Pas de Modification 4.0 International. 


\section{Indiennes : un tissu à la conquête du monde}

\section{Nouveau Centre des indiennes au Musée national suisse - Château de Prangins}

$\mathrm{L}$

es indiennes sont des tissus de coton teint, peint ou imprimé, produits à l'origine en Inde, d'où elles tirent leur nom. Dès les premiers en Asie et en Afrique. À la suite des grandes découvertes et de l'ouverture de nouvelles voies maritimes, les indiennes pénétrèrent en Europe. Leur succès fut tel, y compris en Suisse, qu'il suscita des imitations, des interdictions, de l'espionnage commercial, de la contrebande et beaucoup de convoitises.

En 2016, le Musée national suisse put acquérir un ensemble significatif de 150 indiennes provenant d'une collection de renommée internationale, celle de l'expert en textiles anciens Xavier Petitcol. Cette sélection vint compléter les toiles et les habits d'indienne principalement suisses que le Musée national conservait déjà. Avec la collection Petitcol, il était désormais possible de raconter l'histoire passionnante de ces tissus de façon beaucoup plus exhaustive et précise grâce à des toiles bien documentées et datées. Cette acquisition fit, deux ans plus tard, l'objet d'une grande exposition au château de Prangins (22 avril-14 octobre 2018) (Fig. XLI et XIII, cahier couleur). Accompagnée d'une publication de référence ${ }^{1}$, elle connut un succès retentissant. Celui-ci s'expliqua notamment par le fait

1. Bieri Thomson, Jacqué B., Jacqué J., Petitcol, 2018.

95 Helen Bieri Thomson, «Indiennes : un tissu à la conquête du monde. Nouveau Centre des indiennes au Musée national suisse - Château de Prangins ", Artefact, 12, 2020, p. 279-284. 
que les Suisses découvraient un pan de leur histoire à la fois important et passionnant, mais largement méconnu.

En effet, rappelons ici que l'indiennage constitue véritablement une grande success-story suisse. L'industrie cotonnière fut un des principaux moteurs économiques du pays au XviII ${ }^{\mathrm{e}}$ siècle et l'impression des cotonnades fit vivre des régions entières, particulièrement en Suisse romande, le long de l'arc jurassien, comme à Genève ou à Neuchâtel. De plus, l'indiennage inséra la Suisse au cœur de l'économie mondiale. Par ailleurs, les Suisses furent nombreux à exporter leur savoir-faire ou leurs capitaux en s'impliquant dans des entreprises à l'étranger. Enfin, certains d'entre eux jouèrent également un rôle dans le commerce triangulaire et la traite des Noirs, tous deux intimement liés à l'histoire des indiennes.

En raison de son histoire particulière, le château de Prangins se prêtait particulièrement bien à une évocation de l'épopée des indiennes. Louis Guiguer qui fit construire le château dans les années 1730 appartenait au grand réseau des banquiers, financiers et négociants protestants qui, entre autres, contrôlait les circuits d'importation des denrées coloniales et était impliqué dans le commerce des indiennes. Par ailleurs, des toiles peintes sont mentionnées dans les inventaires de biens des barons de Prangins. On les utilisait comme tentures sur les murs, comme rideaux aux fenêtres, elles garnissaient le mobilier. Les enfants Guiguer, par exemple, dormaient dans des lits d'indiennes. La restitution de l'appartement de réception du château de Prangins, réalisée en 2013, illustre la place qu'occupaient ces étoffes dans un intérieur noble au siècle des Lumières².

Résumons : une collection de premier plan, un chapitre capital de l'histoire suisse, des liens tangibles avec le lieu dans lequel se trouvent les visiteurs, autant de raisons, nous sembla-t-il, de pérenniser ce contenu tout en l'approfondissant et en l'élargissant. Concrètement, en janvier 2021, le Musée national suisse - Château de Prangins inaugurera le premier centre entièrement dédié aux indiennes. Il se composera d'une exposition permanente, d'un espace d'étude, ainsi que d'une offre d'ateliers de teinture végétale.

2. Bieri Thomson, 2013. 


\section{Indiennes : un tissu à la conquête du monde}

La nouvelle exposition permanente propose de croiser histoire locale et globale et invitera le public à un tour du monde en suivant la trajectoire des indiennes et en s'interrogeant sur l'impact qu'elles ont eu dans les différents centres de production et de consommation sur quatre continents ${ }^{3}$. L'itinéraire de visite qui emmènera le public de l'Inde au Brésil, en passant par la Suisse, la France et l'Afrique de l'Ouest, a été imaginé pour faire comprendre le commerce triangulaire - qui voit des tissus de coton marchandés contre des êtres humains, lesquels sont à leur tour échangés contre des denrées coloniales - et ses implications. Tout au long du parcours, la présence et le rôle des Suisses seront soulignés. En effet, en maints endroits du monde, à divers échelons et dans différentes fonctions, des Suisses contribuèrent, directement ou indirectement, à écrire l'histoire de ces étoffes. En fin de visite, le public aura saisi, nous l'espérons, pourquoi les historiens considèrent les indiennes comme l'un des premiers produits mondialisés de tous les temps.

Pour les chapitres consacrés à l'Amérique et à l'Afrique, des collaborations avec des spécialistes sur place s'avérèrent indispensables. Par exemple, grâce à un partenariat avec ICOM (International Committee of Museums), le Musée national suisse - Château de Prangins put participer à un projet pilote de séjour d'étude. Pendant deux semaines en juillet 2019, il accueillit Mohamadou Moustapha Dieye, historien et spécialiste des textiles africains qui travaille dans l'un des plus importants musées du Sénégal, le musée Théodore-Monod d'art africain. Avec son expertise, l'équipe de projet conçut un module consacré au rôle joué par les indiennes en Afrique de l'Ouest. Le regard porté par ce collègue sur le concept global

\footnotetext{
3. Un comité scientifique a accompagné la phase conceptuelle du projet et validé le contenu. Il était composé de Gilles Forster, ancien assistant à l'université de Genève ; Aziza Gril-Mariotte, maitre de conférences à l'université de Haute-Alsace ; André Holenstein, professeur à l'université de Berne ; Bernard Jacqué, historien des arts industriels et ancien maître de conférences à l'université de Haute-Alsace ; Jacqueline Jacqué, conservateur honoraire au musée de l'Impression sur Etoffes de Mulhouse ; Lisa Laurenti, conservatrice adjointe au musée d'art et d'histoire de Neuchâtel ; Liliane Mottu Weber, professeur titulaire émérite de l'université de Genève ; Giorgio Riello, professeur à la Warwick University ; Béatrice Veyrassat, ancienne maître d'enseignement et de recherche à l'université de Genève.
} 
de l'exposition, sur des objets issus des collections du Musée national et, de manière générale, sur nos références en matière d'histoire culturelle, enrichit considérablement notre propos.

En ce qui concerne le chapitre dédié à la Suisse, il fallut surmonter un défi de taille : si textes et archives permettent d'envisager l'ampleur des activités générées par les indiennes dans ce pays au XVIII ${ }^{\mathrm{e}}$ siècle, peu de toiles ont survécu par rapport au nombre de manufactures. De fait, l'industrie des indiennes nécessitait d'importants capitaux et le marché suisse offrait trop peu de débouchés. C'est pourquoi les fabricants privilégièrent l'exportation, principalement vers la France, pays voisin très peuplé jouissant d'un bon niveau de vie.

Comment pallier le manque de toiles ? Deux options furent retenues. D’une part, montrer au public des exemples des nombreuses traces matérielles laissées par l'industrie des indiennes dans les arts visuels et les archives (Fig. XLIII et XLIV, cahier couleur) : vues de manufactures, portraits d'indienneurs, descriptions de fabriques par des voyageurs, mentions dans les inventaires de biens, demandes d'autorisation pour installer une manufacture, ordonnance sur les fabriques et autres règlements. D'autre part, créer un multimédia qui propose une vue d'ensemble inédite de la production des indiennes en Suisse au XvıII ${ }^{\mathrm{e}}$ siècle grâce à la compilation d'informations issues d'études souvent difficilement accessibles au grand public. Ce dispositif non exhaustif rendra vie à ce qui a le plus souvent disparu et remettra en lumière des centres de production très importants, mais rarement évoqués comme Zurich ou Berne.

\section{Espace d'étude}

Un espace d'étude permettra de prolonger la visite de l'exposition permanente. Elle montrera d'autres toiles tirées du fonds de Xavier Petitcol, sélection qui changera régulièrement. Grâce à des supports multimédias interactifs, le public pourra se familiariser avec les techniques de fabrication, l'iconographie des toiles et les productions de diverses manufactures. Il aura la possibilité de comparer des étoffes, de déceler leurs particularités, de reconnaître les différents procédés d'impression, et ce en fonction de ses propres centres d'intérêt. De plus, un poste de consultation permettra de sonder la base de données des collections du Musée national suisse, rendant ainsi possibles des rapprochements entre indiennes et autres types d'objets, par exemple des soieries ou des papiers peints. 


\section{Plantes et teintures}

Un riche programme de cours alternant théorie et exercices pratiques complétera le discours proposé dans les salles d'exposition et permettra une appréhension plus sensorielle et tactile de l'univers des indiennes. Les ateliers donneront la possibilité de tester différentes étapes du processus de teinture végétale : cueillettes de plantes, essais de grand teint et petit teint, teinture d'indigo, techniques d'impression, initiation à l'extraction de fibres textiles. Ils se dérouleront en partie dans le jardin potager historique du château de Prangins où sont cultivées quelque 200 variétés de fruits et légumes anciens, mais aussi de plantes industrielles dont des espèces tinctoriales comme la garance, la gaude, l'indigo et le carthame des teinturiers. Faisant des allers-retours entre le jardin, l'exposition et l'atelier, les participants auront tout loisir de confronter leurs essais aux réalisations du XVIII ${ }^{\mathrm{e}}$ siècle et d'appréhender ainsi les subtilités de la fabrication d'une indienne.

\section{En résumé}

Avec son nouveau Centre des indiennes, le château de Prangins offrira une approche globale et transversale d'un passionnant sujet mêlant histoire culturelle, histoire économique et histoire de l'art. En traversant les salles richement décorées et ornées d'indiennes de l'appartement de réception du XVIII ${ }^{\mathrm{e}}$ siècle, le visiteur découvrira la face séduisante de ces étoffes dans leur variante luxueuse : vivacité des couleurs, exotisme des motifs, confort et légèreté. En pénétrant dans l'exposition permanente Indiennes : un tissu à la conquête $d u$ monde, il percevra les facettes nuancées, et parfois même franchement sombres de la mondialisation à l'époque des Lumières. Dans l'espace d'étude ou en parcourant le jardin potager historique, il prendra conscience de tout le savoir technique que présupposait la fabrication de ces étoffes. Confronté à un discours complexe et polysémique, il repartira, telle est notre ambition, avec des clés de lecture pour mieux comprendre certains enjeux de la globalisation d'hier, mais peut-être aussi d'aujourd'hui.

Helen Bieri Thomson Musée national suisse - Château de Prangins 
Helen Bieri Thomson

\section{Bibliographie}

Bieri Thomson Helen, Jacqué Bernard, Jacqué Jacqueline, Petitcol Xavier (dir.), Indiennes. Un tissu révolutionne le monde!, La Bibliothèque des Arts, Lausanne, 2018.

Bieri Thomson Helen (dir.), Noblesse oblige! La vie de château au XVIIt siècle, 5 Continents, Milan, 2013. 